\title{
Spatial and spectral variability due to Landsat image acquisition date: The impact on image classification
}

\author{
Mike Wulder^ and Steven E. Franklin* \\ ^ Canadian Forest Service, 506 West Burnside Road, Victoria, BC, Canada V8Z 1M5 \\ email: mwulder@nrcan.gc.ca, phone: 250 363-6090, fax: 250 363-0775 \\ * Department of Geography, University of Calgary, Calgary, AB, Canada T2N 1N4
}

\begin{abstract}
Large area land cover mapping with Landsat data is affected by differing image spectral characteristics caused by factors such as solar, radiometric, atmospheric, and phenologic conditions. For mapping land cover, especially forested land cover with single date satellite imagery, acquisition of imagery is ideally undertaken during the peak growing periods during the summer months. Yet, during the summer months cloud cover may preclude the collection of suitable imagery. To enable the collection of imagery to compile a large area coverage, the range of acceptable acquisition dates must be extended from the optimal mid-summer period. The question is how far can the acquisition dates range before the spectral information in the imagery is no longer appropriate for land cover mapping. To address this issue we have classified Landsat imagery representing a range of within year and between year dates and have assessed the variability in spatial features developed and the spectral information contained in the imagery.

To ensure consistent radiometric characteristics between scenes we applied a top of atmosphere radiometric correction and a dark target subtraction. Our data set consists of 7 images. To assess within year variability we obtained 5 images over a range of dates within 1999. To calibrate the within year differences, we obtained images to represent the previous (1998) and following year (2000) during an optimal image acquisition window. Our results have shown that differences in class area are highly variable over the range of image acquisition dates. Spatial metrics also illustrate that the composition of the resultant classification units are also variable. The spectral variability of the within year image set are found to exceed those found for the between year image set.
\end{abstract}

\section{INTRODUCTION}

Land cover mapping objectives have continued to expand to include mosaics of Landsat Thematic Mapper (TM) multitemporal imagery over large geopolitical and ecological areas [1]. Understanding and developing methods of handling a spatially-changing spectral response pattern, caused by solar, radiometric, atmospheric, phenologic, and other differences captured in imagery, represents a significant challenge for the remote sensing community in this application [2]. Unfortunately, methods of image analysis in support of such large-area TM mapping of land cover are fragmented and poorly developed [3]. Depending on the purpose of the classification, the skill of the analysts, and the tools available, methods used have ranged from relatively simple unsupervised clustering algorithms to more comple $\mathrm{x}$ decisionrule based classifiers that employ aspects of spatial reasoning and set theory. In support of Earth Observation for Sustainable Development of Forests (EOSD) [4] and the need to develop a national land cover classification product [5], understanding and resolving the many technical difficulties in repetitive and robust large-area classification must be tested and assessed prior to implementation.

We reviewed numerous possible options for the classification algorithm/approach for EOSD Land Cover [3] and determined that, with consideration of the project possibilities and constraints, a Hyper-Clustering and Labelling Procedure $(H C L P)$, is an appropriate approach. HCLP is an unsupervised technique generally recommended in areas where little is known of the class structure or where training (field) data are scarce or impractical to acquire (e.g., $[6,7,8]$ ). It has been suggested that the hyperclusters require relatively little manual effort (thereby limiting field and ancillary data needs [1]). It is also apparent that the tasks are generally wellunderstood and easily implemented in commercially - or publicly-available image processing systems. The procedure appears to have the potential to be robust and repeatable.

A test is required of the $\mathrm{K}$-means clustering algorithm commonly employed within the general HCLP process. The goal is to determine the ability of an HCLP to handle the differences in vegetation phenology that accompany seasonal imagery. For example, it is well known that the Kmeans algorithm is influenced by four major characteristics [9]:

1. the number of cluster centres specified,

2. the choice of initial cluster centres,

3. the order in which the samples are taken, and,

4. the geometrical properties of the data.

Proceeding of the International Geoscience and Remote Sensing Symposium (IGARSS) and $24^{\text {th }}$ Symposium of the Canadian Remote Sensing Society, June 24-28, Toronto, Canada. 
Are these influences small enough that imagery acquired at different times of the year in a large-area mosaic can be processed with a virtually-automatic method? Could decisions taken during cluster merging by an image analyst reduce these influences? More generally, anniversary or multiple year imagery will likely be employed in large-area TM mosaics without physically-based radiometric processing (i.e. with only image normalization or preliminary radiometric correction that does not deal with atmospheric effects); what is the influence of the scene-dependent factors on the ability of the hyperclustering approach to generate the land cover classes required over large spatially-variable image mosaics? To help address this question, we devised an experiment involving seven different Landsat images acquired over one growing season and three years (Table 1) in the region of Prince George, British Columbia, Canada:

1. Classify a large region (approximately 100000 square kilometres, a single Landsat TM scene) in several image dates to simulate the expected variability that will be contained in large-area Landsat TM mosaics such as are available in Canada (e.g., the Centre for Topographic Information (CTI) satellite image database) [10];

2. Determine the range and stability of the radiometrically normalized input digital numbers by examining representative features in some of the broad classes proposed for EOSD Land Cover;

3. Statistically examine the stability of the classes that emerge after hyperclustering the data sets using both per-pixel and 'area-based' assessments;

4. Visually interpret the classes and spatial configuration of classes to lend confidence in the statistical comparisons; and assess qualitatively the expected effects of clouds and other changes in the imagery on the HCLP with an interpretation of class stability and persistence over time.

\section{METHODS \\ A. Image Acquisition and Processing}

The available images acquired for the Prince George study area (Path 49, Row 22) are listed in Table 1. Clouds were a problem in most of the imagery; only the August 3, 1999 image was reasonably cloud-free with some haze. This image was selected as the 'master' image for the tests in this paper; the 'master' against which the others could be compared.
TABLE 1

TM IMAGERY SELECTED FOR TESTS IN THE PRINCE

\begin{tabular}{|c|l|c|}
\hline Satellite & \multicolumn{1}{|c|}{ Date } & $\begin{array}{c}\text { Solar } \\
\text { Zenith } \\
\text { (degrees) }\end{array}$ \\
\hline Landsat 5 & July 31, 1998 & 51 \\
\hline Landsat 5 & April 29, 1999 & 47 \\
\hline Landsat 5 & July 18, 1999 & 53 \\
\hline Landsat 5 & August 3, 1999 & 50 \\
\hline Landsat 7 & September 12, 1999 & 38.6 \\
\hline Landsat 5 & September 20, 1999 & 35 \\
\hline Landsat 7 & June 26, 2000 & 56.5 \\
\hline
\end{tabular}

B. Georadiometric Processing

The August 3, 1999 image was orthorectified using 15 GCPs identified on the imagery and in the 1:50000 scale topographic maps available for the area. The RMS error in pixel units in this registration was 1.33 in the $\mathrm{x}$-direction and 1.18 in the $y$-direction. This image was used as the master for the correction of the remaining six scenes. Cubic convolution resampling was used to output the geocoded data. Top-of-Atmosphere radiometric correction and a dark-object subtraction procedure was implemented for an atmospheric correction on each of the seven image scenes.

\section{Hyper-Clustering and Labelling Procedure (HCLP)}

We implemented the K-means algorithm within the following five-step HCLP:

1. Generation of a cloud mask using interactivethresholds for each image;

2. K-means clustering after 12 iterations (the maximum allowable in the formulation available) and 50 clusters requested (this amount was thought to represent a reasonable compromise between the number of clusters that could be managed reasonably by a human interpreter in the following step and the maximum number - more than 240 - that can be generated in the available code);

3. Cluster merging based on Bhattacharrya distance separability measures and visual cues in the imagery for known class features (e.g., grassy areas at the Prince George Airport, forest cover types available in the GIS, lakes, etc.);

4. Recode of clusters to an 8 class scheme (snow/ice, water, dense conifer, open conifer, deciduous/shrub, shrub/grass, grass, exposed soil, cultural features);

5. Export of recoded class scheme to GIS map creation and display software. 
The classification analysis was conducted in three steps:

1. Area Analysis - this involved the comparison of total class areas in the full scene and in several selected subareas. The maximum and minimum differences, expressed as percent compared with the 'master', were compiled in areas with and without the cloud masks.

2. Correspondence Analysis - this involved a pixel-by-pixel comparison of the image classes for the entire TM scene. A confusion matrix and the kappa coefficient of agreement were used to summarize the agreement between classes on the 'master' and the six other images on a pixel-by-pixel basis.

3. Spatial Indices Analysis - this involved the computation and comparison of the following representative landscape metrics for selected subareas: class area (ha), number of patches, mean patch size (ha), edge density, mean proximity index). These are presented as percent difference between the 'master' image and the remaining six images in the database.

\section{RESULTS AND DISCUSSION}

We report a test of the sensitivity of the $\mathrm{K}$ means clustering algorithm, as part of a five-step Hyper-Clustering and Labelling (HCLP) procedure, to issues related to seasonality, cloud, ice/snow, and changing vegetation phenology. Large differences in the classes resulting from HCLP classification of seven Landsat TM images acquired over three years and in five different months of the Prince George area were interpreted:

- Visually, the different imagery contained large and variable amounts of cloud, haze, ice and snow, which affected the initial clustering sequence and results. In one case, clouds obscured the city of Prince George, and no urban/cultural/road class could subsequently be distinguished in the hyperclusters.

- $\quad$ The selected TM images displayed differences in general land cover condition that would be consistent with the seasonal changes; i.e., in vegetated areas, the June, July, and August scenes showed higher 'greenness' in normal colour composites, and the later fall and early spring scenes showed more 'brownness'.

- Several features changed reflectance dramatically as a consequence of the formation and melting of ice. The recently cut areas appeared to contain significant variability related to the presence of snow in the April 29, 1999 image; but a reasonably stable spectral response pattern existed for these features in the visible bands in the other scenes. There was a tendency for increased DN values to occur in the September imagery; perhaps related to the senescence of broad-leaved species in the clearcuts. The older cuts appeared to have a diminished 'snow-effect' in the April 29, 1999 imagery compared to the recent cuts, and also to contain less variability from scene to scene in general. There was a tendency for the green band to increase in $\mathrm{DN}$ values to a greater extent than the other two bands in the September imagery. In the June 26, 2000 scene, the green band was much higher than either the blue or red bands; this was interpreted as indicating a higher shrub content and early broad-leaf emergence.

- The conifer feature selected appeared stable in the July and August scenes; no more than one DN value change. In September, the DN values increased considerably (almost by a factor of two) in the blue and red bands, and by a factor of three in the green band. The June 26, 2000 image showed a different pattern; greater values in the green than the blue and red. The normal pattern appeared to be for decreasing $\mathrm{DN}$ values in the blue, green, then red. The 29 April 1999 image contained the highest DN values, as expected: the 'snow-effect', even though the area did not contain visible amounts of snow in the stand selected for this study.

- The open wetland in the July and August scenes displayed values very similar to those obtained in the conifer forest stand and a similar seasonal pattern was apparent. Later, in September, the open wetland showed higher DN values than in the June, July, and August imagery. The pattern between the red and green bands observed in the conifer stand was reversed for the open wetland, however.

- Deciduous forest DN values increased as the season progressed; the lowest values were observed in June, and the highest (apart from the snow-effect ) were observed in September. The pattern between the red and green bands resembled that of the open wetland more so than the conifer stand, with 
a predictable high green peak in June and September. In July and August, green and red reflectance were more equal.

- The kappa coefficient of agreement suggested that the correspondence was at best moderate between the 'master' and the July 18, 1999 and June 26, 2000 HCLP classification product classes; the other four only weakly corresponded. These results suggested very low persistence and stability of most vegetation classes.

- In two selected subareas (and four others not discussed but mentioned here for completeness), we found that spatial indices such as the number of patches, edge density, and mean proximity index, varied greatly with different HCLP output maps. The smallest absolute differences, for example, exceeded $4 \%$ in each of the tested spatial metrics across the six HCLP output maps for a relatively undisturbed environment with large patches and no noticeable land cover change.

\section{CONCLUSIONS}

Our results have shown that differences in class area are highly variable over the analysed range of image acquisition dates. Spatial metrics also illustrated that the composition of the resultant classification units are also variable. The spectral variability of the within year image set are found to exceed those found for the between year image set. This is an important finding as it indicates that offyear imagery may be preferred to off-season imagery. As a result, if image classification is your only aim, off-year imagery from the same season is preferred over same year off-season imagery. While off-year / on-season imagery may be preferred for classification objectives, potential conflicts may arise for fixed year studies of image change detection. This research indicates the need to carefully select imagery for large area land cover mapping projects. When selecting imagery it is important to remain as close to peak photosynthetic activity as possible. Due to issues such as cloud cover, imagery will often not be available during the optimal acquisition period requiring users to consider the implications of the selection and inclusion of an off-season image in the large area classification.

\section{ACKNOWLEDGEMENTS}

This research was funded by the Canadian Forest Service and the Natural Sciences and Engineering Research Council of Canada. We are grateful to Mr Tom McCaffrey for his efforts in processing the imagery.

\section{REFERENCES}

[1] C. G. Homer, R. D. Ramsey, T. C. Edwards, Jr., and A. Falconer, "Landscape cover-type modeling using a multiscene Thematic Mapper mosaic," Photogrammetric Engineering and Remote Sensing, vol. 63, pp. 59-67, 1997.

[2] J. Cihlar, "Land cover mapping of large areas from satellites: status and research priorities," Int. J. Remote Sensing, vol. 21 pp. 1093-1114, 2000.

[3] S. Franklin, and M. Wulder, "Remote sensing methods in high spatial resolution satellite data land cover classification of large areas," Progress in Physical Geography, in press.

[4] J. Wood, M. Gillis, D. Goodenough, R. Hall, D. Leckie, J. Luther, and M. Wulder, "Earth Observation for Sustainable Development of Forests (EOSD): Project Overview," Proc. International Geoscience and Remote Sensing Symposium 2002, Toronto, ON, Canada, 2002.

[5] M. Wulder, "Mapping the land cover of the forested area of Canada," Proc. International Geoscience and Remote Sensing Symposium 2002, Toronto, ON, Canada. 2002.

[6] S. S. Talbot, and C. J. Markon, "Intermediate-scale vegetation mapping of Innoko National Wildlife Refuge, Alaska, using Landsat MSS digital data," Photogrammetric Engineering and Remote Sensing, vol. 54 pp. $377-383,1988$.

[7] M. E. Bauer, T. E. Burk, A. R. Ek, P. R. Coppin, S. D. Lime, T . A. Walsh, D. K. Walters, W. Befort, and D. F. Heinzen, "Satellite inventory of Minnesota forest resources," Photogrammetric Engineering and Remote Sensing, vol. 60, pp.287-298, 1994.

[8] D. M. Debinski, K. Kindscher, and M. E. Jakubauskas, "A remote sensing and GIS-based model of habitats and biodiversity in the Greater Yellowstone ecosystem," Int. J. Remote Sensing, vol. 20, pp. 3281-3291, 1999.

[9] J. T. Tou, and R. C. Gonzalez, Pattern Recognition Principles, Reading, MA, Addison-Wesley, 1974.

[10] M. Wulder, E. Loubier, and D. Richardson, “ A Landsat -7 ETM+ orthoimage coverage of Canada”, Can. J. Remote Sensing, in review. 